\title{
A importância dos exames neurológico e radiográfico no diagnóstico de lesões cervicais em equino: relato de caso
}

\author{
[Fundamentals of neurological and radiographic examination on diagnosis of cervical lesions \\ of a horse: case report] \\ G.G. Rivera, G.M. Bueno, V.B. Paula, M.Q.P. Santo, A. Matsui, A. Escoba, R.O. Vasconcelos, \\ J.C. Canola, P.A. Canola, J.C. Lacerda-Neto \\ Universidade Estadual Paulista - Unesp - Jaboticabal, SP
}

\begin{abstract}
RESUMO
As lesões vertebrais cervicais são relativamente comuns nos equinos. No entanto, são poucas as descrições a respeito desse tema na literatura. Acredita-se que esse fato se deva, em parte, às limitações relacionadas ao diagnóstico. Em virtude de um traumatismo cervical, o animal pode apresentar sinais clínicos de paresia e paraplegia até tetraparesia ou tetraplegia, que podem culminar em estado comatoso e, eventualmente, em morte. Esses sinais clínicos podem ser detectados no exame físico, porém, quando associado à imaginologia (avaliação radiográfica), permite a obtenção de diagnóstico mais preciso da lesão. Consequentemente, um prognóstico mais adequado pode ser estabelecido para o paciente. Em face do exposto, apresenta-se o caso de uma égua Mangalarga, de quatro anos de idade, com sinais de tetraplegia pós-trauma, aumento de tônus muscular e paralisia espástica, com hiperreflexia, nos quatros membros. Por meio dos exames neurológicos realizados na paciente, em associação com as imagens radiográficas convencionais e contrastadas, foi possível estabelecer o diagnóstico do quadro. $\mathrm{O}$ animal apresentava lesões envolvendo as duas primeiras vértebras cervicais, atlas $\left(\mathrm{C}_{1}\right)$ e áxis $\left(\mathrm{C}_{2}\right)$, e a quinta e a sexta vértebra $\left(\mathrm{C}_{5}-\mathrm{C}_{6}\right)$, destacando fratura de $\mathrm{C}_{1}$, deslocamento do dente de $\mathrm{C}_{2}$ e subluxação entre $\mathrm{C}_{5}$ e $\mathrm{C}_{6}$, com compressão ventrodorsal da medula espinhal. Confirmadas as suspeitas neurológicas e mediante prognóstico desfavorável, o animal foi submetido à eutanásia, com consentimento do proprietário. As lesões observadas nas imagens radiográficas foram confrontadas e confirmadas na necropsia.
\end{abstract}

Palavras-chave: cavalo, compressão medular, fratura, imaginologia, pescoço

\begin{abstract}
Cervical vertebral lesions are relatively common in equine patients, but its diagnosis seems challenging for the clinician. Clinical signs most commonly associated with this clinical feature are paresis, paraplegia, tetraparesis, tetraplegia, which may lead the patient to a comatose state, and even death. Fortunately, these clinical signs can be perceived during physical examination. All of which, alongside imaging techniques, may allow a precise diagnose of the location of lesions and prediction of a prognosis for the patient. We present a case report of a Mangalarga mare, with four years of age, presenting signs of post-traumatic tetraplegia with spastic paralysis with increased muscular tonus and hyperreflexia in all four limbs. A thorough neurological examination of the patient associated with conventional and contrast medium radiographic imaging of the cervical region identified lesions at the atlas $\left(C_{1}\right)$ and axis $\left(C_{2}\right.$, and fifth $\left(C_{5}\right)$ and sixth $\left(C_{6}\right)$ cervical vertebrae. Fracture of the atlas vertebrae, dislocation of the axis' dens as well as subluxation of $C_{5}-C_{6}$ causing ventrodorsal medullary compression of the spinal cord were diagnosed radiographically. Due to the extent of the lesions the animal was euthanatized with the owner's consent. All lesions identified radiographically were posteriorly confirmed during post-mortem examination.
\end{abstract}

Keywords: equine, fracture, imaging, medullary compression, neck

Recebido em 15 de setembro de 2016

Aceito em 3 de dezembro de 2016

E-mail: gabigrivera@hotmail.com 


\section{INTRODUÇÃO}

Apesar da alta incidência dos casos de traumatismo envolvendo o esqueleto axial nos equinos, estes ainda são relativamente pouco diagnosticados e relatados (Silver, 2012). As manifestações clínicas decorrentes do trauma geralmente se traduzem em sinais de tetraparesia e tetraplegia (Lin et al., 2011), em comprometimento funcional severo e até em óbito do paciente (Silver, 2002).

As lesões cervicais comumente são decorrentes de concussões e contusões por queda ou colisão contra objetos estáticos de grandes dimensões. A ocorrência é maior em animais mais jovens, especialmente na porção cervical caudal e torácica caudal do esqueleto axial (Matthews, 2000).

Lesões na medula espinhal causam diversos graus de debilidade, ataxia, alterações nociceptivas e do sistema nervoso autônomo. A presença e a gravidade dos sinais clínicos dependem dos seguintes fatores: localização, extensão, profundidade da lesão no segmento da medula espinhal e acometimento da substância cinzenta da medula espinhal (Riet-Correa et al., 2002).

As radiografias convencionais e contrastadas (mielografia) são de extrema importância para a avaliação de injúrias na coluna vertebral, além da identificação de compressões na medula espinhal (Hudson e Mayhew, 2005). O exame mielográfico é de grande valia na observação, localização e determinação da gravidade de compressão medular. Quando presentes, a tomografia computadorizada (CT) e a ressonância magnética (MRI) podem auxiliar na identificação de lesões envolvendo diretamente a medula espinhal (Biervliet et al., 2004). É importante que as fraturas vertebrais sejam incluídas na lista de diagnóstico diferencial em animais em decúbito permanente (Borges et al., 2003).

O tratamento das lesões vertebrais objetiva preservar o tecido nervoso, minimizar os eventos inflamatórios do trauma e reduzir a peroxidação lipídica da membrana, a hemorragia e o edema locais. O uso de anti-inflamatório esteroidal tem como objetivo a manutenção da concentração glicêmica e o equilíbrio eletrolítico, a redução da disseminação de lesões morfológicas, a preservação da condução axônica e a atividade reflexa. O dimetilsulfóxido (DMSO) também é utilizado com o intuito de preservar o fluxo sanguíneo no tecido cerebral e na medula espinhal. Além disso, acredita-se que promova vasodilatação local, minimize o edema local, a agregação plaquetária e a produção de radicais livres. Os anti-inflamatórios não esteroidais têm função de controlar a inflamação pós-traumática, promovendo maior conforto ao paciente (Matthews, 2000).

É essencial reunir todos os achados clínicos, laboratoriais e os exames de imagem diante de um quadro clínico inespecífico, para a obtenção de um diagnóstico mais fidedigno.

Apesar de as lesões vertebrais serem relativamente frequentes nos equinos, notou-se uma escassez de relatos a respeito do tema, principalmente em relação às minúcias relacionadas aos aspectos clínicos do quadro e os recursos e meios diagnósticos utilizados. Em decorrência disso, buscou-se correlacionar os sinais clínicos de um paciente da espécie equina, com suspeita de lesão envolvendo a região cervical, com os achados radiográficos, visando determinar as consequências da lesão para o paciente e, por conseguinte, estabelecer o prognóstico mais apropriado para o caso em questão.

\section{CASUÍSTICA}

Um equino da raça Mangalarga, fêmea, com quatro anos de idade e $400 \mathrm{~kg}$ de peso corporal, foi encaminhada ao Hospital Veterinário (HV) com histórico de tetraplegia com quatro dias de evolução. Segundo o proprietário, a égua, ao estirar e empinar, enquanto selada e amarrada, girou sobre os membros pélvicos e colidiu a região dorsal da coluna vertebral, a crista nucal e a crista sagital externa do crânio ao solo. Depois do acidente, o animal manteve-se alerta, ficou em posição quadrupedal por duas horas. Após este período, o animal adotou decúbito permanente, alternando da postura esternal para a lateral. $\mathrm{Na}$ propriedade, utilizaram-se diversas medicações, como dexametasona $(0,1 \mathrm{mg} / \mathrm{kg}$, primeira aplicação; $0,08 \mathrm{mg} / \mathrm{kg}$, segunda aplicação; $0,07 \mathrm{mg} / \mathrm{kg}$, terceira aplicação), uma vez ao dia, durante três dias, fenilbutazona $(2,2 \mathrm{mg} / \mathrm{kg})$, uma vez ao dia, 
durante dois dias, e manitol $(0,25 \mathrm{mg} / \mathrm{kg})$, em duas aplicações, em intervalo de 12 horas. Durante os quatro dias em que esteve na propriedade, o animal era mantido em posição quadrupedal, por meio de talha manual, por um período de seis horas/dia. A égua foi encaminhada para o HV no quarto dia póstrauma. $\mathrm{Na}$ admissão, não aparentava alterações comportamentais evidentes, porém mantinha-se em decúbito lateral permanente. No exame físico geral, auscultou-se discreta crepitação pulmonar bilateral. No exame neurológico, observou-se amplitude limitada de movimentação do pescoço, dor à palpação da região cervical, tônus muscular aumentado e paralisia espástica nos quatros membros e flacidez da cauda. Os nervos cranianos e o reflexo cutâneo do tronco estavam preservados. Em contrapartida, o reflexo patelar apresentava-se aumentado. Na palpação retal, constatou-se aumento de volume da vesícula urinária, a qual foi submetida à cateterização e a posterior esvaziamento. Nos exames hematológicos de admissão, constatou-se anemia (6.290 milhões $\left./ \mathrm{mm}^{3}\right)$ e leucopenia $\left(5.200 \mathrm{mil} / \mathrm{mm}^{3}\right)$ discretas. No exame bioquímico, detectou-se aumento das enzimas asparato transaminase (AST) (628UI/L), gamaglutamiltranferase (GGT) (15UI/L), além de hipoalbuminemia $(2,45 \mathrm{~g} / \mathrm{dL})$.

Como tratamento inicial, foi instituído dexametasona $(0,08 \mathrm{mg} / \mathrm{kg}$, primeira aplicação; $0,07 \mathrm{mg} / \mathrm{kg}$ segunda e terceira aplicações), uma vez ao dia, durante três dias, flunixinameglumina $(0,25 \mathrm{mg} / \mathrm{kg})$, três vezes ao dia, durante quatro dias, dimetilsulfóxido (DMSO) (1g/kg), uma vez ao dia, durante quatro dias, e oxitetraciclina $(10 \mathrm{mg} / \mathrm{kg})$ diluída em solução fisiológica a $10 \%$, a cada 48 horas, em duas aplicações. Os medicamentos preconizados foram administrados por via intravenosa. No $10^{\circ}$ dia pós-trauma, repetiram-se os exames laboratoriais, comprovando-se leucopenia com desvio à esquerda $\left(12.100 \mathrm{mil} / \mathrm{mm}^{3}\right)$ e hiperfibrinoginemia $(1,0 \mathrm{~g} / \mathrm{L})$. No exame bioquímico, notaram-se alterações relevantes em comparação ao exame admissional, em relação aos valores das enzimas AST (557UI/L), fosfatase alcalina (431UI/L) e GGT (22UI/L), além do aumento da ureia $(76 \mathrm{mg} / \mathrm{dL})$ e creatinina $(4 \mathrm{mg} / \mathrm{dL})$.

Clinicamente, notou-se acentuada dificuldade respiratória, com crepitação pulmonar bilateral. Com o intuito de confirmar a suspeita clínica de lesões traumáticas em coluna cervical, o paciente foi encaminhado ao Setor de Diagnóstico por Imagem do HV. Para a realização das projeções radiográficas, o paciente foi submetido à anestesia geral. Como medicação pré-anestésica, foi utilizada xilazina $2 \%(0,2 \mathrm{mg} / \mathrm{kg})$; na indução, tiopental sódico $5 \%(5,0 \mathrm{mg} / \mathrm{kg})$, e a manutenção anestésica geral foi mantida com infusão de tiopental sódico $5 \% \quad(5 \mathrm{mg} / \mathrm{kg})$ e isoflurano. Inicialmente, foram realizadas as projeções radiográficas convencionais e, posteriormente, as séries contrastadas, com administração de contraste não iônico. As projeções laterais da coluna cervical foram realizadas antes e após a mielografia, com o pescoço do paciente em posição neutra, flexionada e estendida. No exame radiológico convencional, foi visualizada redução do espaço entre o corpo do atlas $\left(C_{1}\right)$ e o processo espinhoso do áxis $\left(\mathrm{C}_{2}\right)$. A densidade óssea de $\mathrm{C}_{1}$ apresentava-se mais acentuada em relação ao normal (em razão de fratura compressiva) e um fragmento ósseo dessa vértebra localizava-se na região da fóvea articular caudal à fóvea do dente de $\mathrm{C}_{2}$. Nessa região, a face proximal do dente de $\mathrm{C}_{2}$ estava discretamente desviada no sentido anti-horário (Fig. 1-A). Após a obtenção das imagens radiográficas convencionais, realizou-se a punção entre $\mathrm{C}_{1}-\mathrm{C}_{2}$, como descrita por Delahunta, Glass e Kent (2015), para a coleta de líquido cefalorraquidiano (LCR) e a realização da série radiográfica contrastada (mielografia). O meio de contraste utilizado foi o iopamidol (Iopamiron $300 \AA$ ), na dose $0,45 \mathrm{~mL} / \mathrm{kg}$. À avaliação do LCR, não foram encontradas alterações dignas de nota. A coluna de contraste, em toda a sua extensão de preenchimento, dorsal e ventral à medula espinhal, apresentava-se irregular pela presença de áreas radioluscentes, provavelmente sugestivas de coágulos sanguíneos nos espaços subaracnoideo e epidural, associados ou não à instalação de mielite (Fig.1-B). Imagens contrastadas provenientes da mielografia caracterizaram, além das lesões verificadas e descritas com base nas imagens convencionais, a parada súbita da coluna de contraste entre a face caudal de $\mathrm{C}_{5}$ e a cranial de $\mathrm{C}_{6}$, com compressão ventrodorsal da medula espinhal. $\mathrm{O}$ espaço intervertebral entre $\mathrm{C}_{5}-\mathrm{C}_{6}$ se encontrava diminuído, as faces articulares entre essas vértebras cervicais apresentavam-se irregulares pela compressão, com consequente fratura e deslocamento dorsal da epífise distal de $\mathrm{C}_{5}$. $\mathrm{O}$ corpo de $\mathrm{C}_{6}$ estava 


\section{Rivera et al.}

deslocado ventralmente em relação ao corpo vertebral de $\mathrm{C}_{5}$. Fragmentos ósseos provenientes de fraturas da face caudal do processo ventral de $\mathrm{C}_{5}$ e do processo transverso de $\mathrm{C}_{6}$ foram evidenciados nesse local (Fig.1-C). Após validação diagnóstica e autorização do proprietário, o animal foi submetido à eutanásia por infusão de cloreto de potássio, pela via intravenosa, durante a anestesia geral. $\mathrm{Na}$ necropsia observou-se pulmão difusamente vermelho-escuro, crepitante e congesto. Os lobos pulmonares direito, além de hipocrepitantes, apresentavam impressões das costelas na porção dorsal (Fig. 1-E). À microscopia, o tecido pulmonar apresentava hemorragia intra-alveolar, congestão difusa do parênquima e infiltrado inflamatório difuso (Fig. 1-G). $\mathrm{Na}$ coluna vertebral, verificou-se a presença de extensas áreas de hemorragia na musculatura, de coágulos cruóricos no espaço epidural e subaracnoideo, subluxação entre $\mathrm{C}_{5}-\mathrm{C}_{6}$, e compressão da medula provocada pelo deslocamento da epífise distal de $\mathrm{C}_{5}$ para a porção ventral do canal medular (Fig. 1-D). Nas regiões torácicas, lombar e sacral, não foram evidenciadas alterações vertebrais e medulares. Observou-se ainda hemorragia subdural no assoalho da caixa craniana, adjacente à cela túnica.

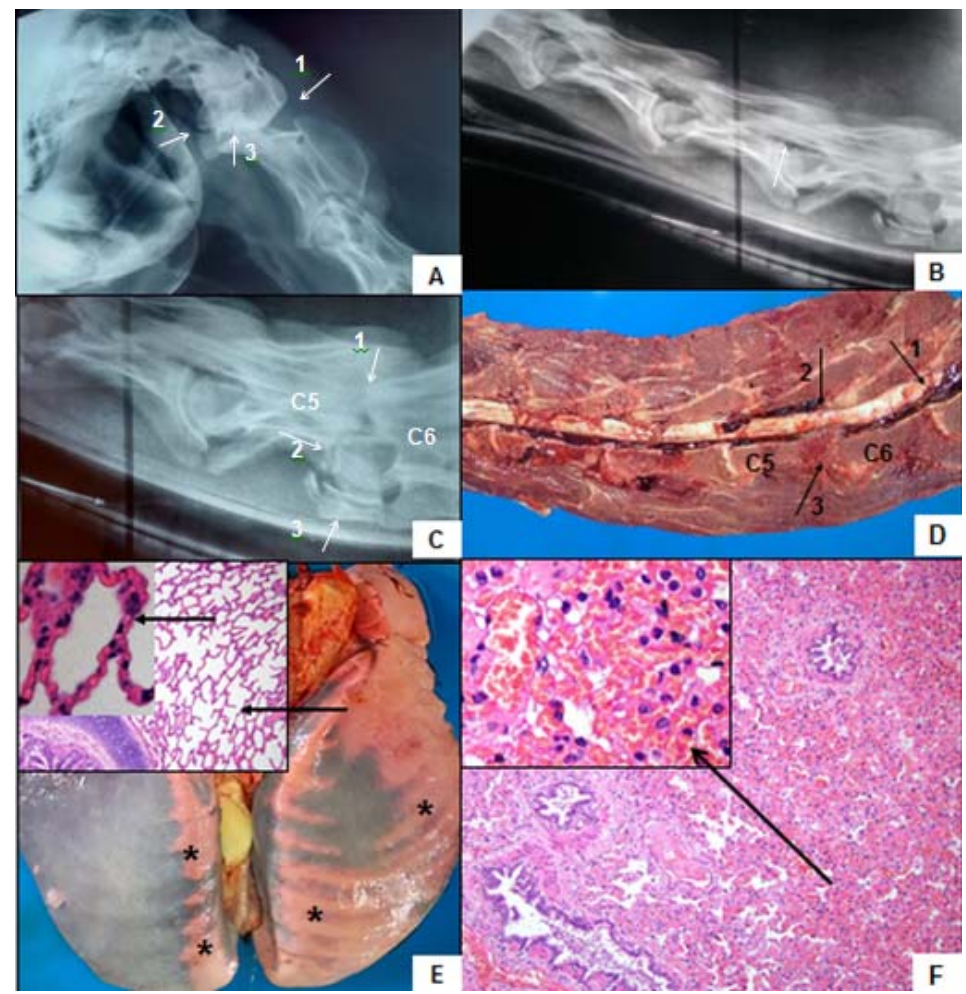

Figura 1. A - Imagem radiográfica simples, em projeção lateral, da região cervical de equino, fêmea, da raça Mangalarga, ilustrando redução de espaço entre as vértebras cervicais atlas $\left(C_{1}\right)$ e o processo espinhoso do áxis $\left(C_{2}\right)$, deslocado do seu eixo normal, com aumento da radiopacidade decorrente de fratura por compressão caudoventral de $\mathrm{C}_{1} ; 2$, presença de fragmento ósseo correspondente à fóvea articular caudal de $\mathrm{C} 1 ; 3$, deslocamento do dente de $\mathrm{C} 2$. $\mathrm{B}$ - Imagem radiográfica contrastada (mielografia), em projeção lateral, da região cervical do mesmo animal, com a presença de área radiopaca irregular da linha de contraste, sugerindo presença de hemorragia no canal medular (seta branca) entre $\mathrm{C}_{4} \mathrm{C}_{5}$. C - 1 Mielografia da região cervical do mesmo animal, em projeção lateral, ilustrando a interrupção da coluna de contraste no segmento $\mathrm{C}_{5}-\mathrm{C}_{6} ; 2$, irregularidade da faceta articular de $\mathrm{C}_{6} ; 3$, fratura do processo articular do processo transverso $\mathrm{C}_{5}-\mathrm{C}_{6}$. D - Peça anatômica da coluna cervical: 1 , áreas com coágulos cruóricos no canal vertebral, localizadas entre $\mathrm{C}_{5}-\mathrm{C}_{6} ; 2$, subluxação com fratura da epífise caudal de $\mathrm{C}_{5} ; 3$, compressão ventrodorsal da medula espinhal entre $\mathrm{C}_{5}-\mathrm{C}_{6}$. E -Necropsia: pulmão ilustrando macroscopicamente a impressão das costelas na porção dorsal dos lobos pulmonares do animal, associada a áreas de consolidação vermelho-escura do parênquima (*;). Detalhe - Fotomicrografia do pulmão, correspondente à área de aspecto normal do órgão. Nota-se congestão dos septos alveolares (hematoxilina e eosina, Obj.10x). F - Histologia: fotomicrografia do pulmão, com presença de hemorrragia intra-alveolar difusa acentuada e congestão difusa moderada (hematoxilina e eosina, Obj.10x). 


\section{DISCUSSÃO E CONCLUSÃO}

Afecções da coluna vertebral são fatores intrínsecos que podem alterar o desempenho dos cavalos em relação ao rendimento esportivo ou até mesmo condenar o animal, como no caso deste relato (Lin et al., 2011). Algumas condições de manejo podem predispor à ocorrência de fraturas vertebrais. A égua em questão era extremamente agitada, segundo o proprietário; uma doma racional e manejos adequados, portanto, poderiam ter evitado o acidente aqui descrito (Borges et al., 2003).

A realização do exame neurológico e suas repetições foram imprescindíveis para predizer a localização das lesões e a evolução da enfermidade. Os recursos de imagens utilizados permitiram evidenciar a lesão localizada na região de vértebras cervicais, com suspeita de compressão medular nos ditos segmentos cervicais e consequente lesão de neurônio motor superior (NMS). Mais especificamente, suspeitava-se de lesão entre $\mathrm{C}_{1}$ e $\mathrm{C}_{4}$ ou nos tratos descendentes do tronco encefálico. No presente relato, tanto os membros pélvicos como os torácicos foram acometidos gravemente. Geralmente, em lesões medulares, os membros pélvicos apresentam sinais mais evidentes pelo fato de os tratos proprioceptivos desses membros estarem localizados mais superficialmente na medula espinhal, quando comparados aos dos membros torácicos. Outra explicação plausível para o fato sugere que a ataxia pode ser mais proeminente nos membros pélvicos devido à maior distância entre eles e o centro de gravidade corporal, o qual se encontra mais próximo aos membros torácicos, além da elevada concentração de NMS na substância cinzenta da intumescência cervical. Quando os membros torácicos e os pélvicos são afetados simultaneamente, fica implícita a presença de lesão na região cervical alta, ou seja, entre $C_{1}$ e $\mathrm{C}_{5}$ (De Lahunta et al., 2015). Alterações clínicas, como presença de tônus muscular aumentado, paralisia espástica, com hiperreflexia, nos quatros membros, são referidas como características de lesão em neurônio motor superior (Riet-Correa et al., 2002).

Mecanismos voluntários e involuntários controlam a funcionalidade da bexiga, que, logo após um trauma medular, torna-se atônica pela atividade reflexa. A retenção urinária é resultado imediato da lesão medular. A distensão vesical ocasiona hiperextensão da bexiga e do músculo detrusor, o que pode retardar o retorno da função vesical (Steffen-Albert e Jeffeeson, 2012). O cateterismo da vesícula urinária intermitente é indicado no tratamento da disfunção vesical. Nesse caso, assim como descrito na literatura, a sondagem vesical permitiu o retorno da função fisiológica do órgão (Moroóka, 2000).

O relaxamento de cauda seguido de incontinência urinária sugere a instalação da síndrome da cauda equina. Nesse caso, as suspeitas de lesões em vértebras lombares, com consequente compressão do canal medular, foi descartada durante a realização da necropsia e nas análises microscópicas. A suspeita da síndrome da cauda equina foi aventada pelo provável traumatismo na região lombar pelo impacto e pela pressão exercida pela sela nessa região, a qual poderia ter causado compressão dinâmica da medula espinhal.

As lesões pulmonares sugerem o desenvolvimento de pneumonia intersticial, embora cuidados profiláticos, como mudança de decúbito constante, tivessem sido realizados. $\mathrm{O}$ fator predisponente para a pneumonia intersticial foi possivelmente atribuído ao escore corporal elevado (7/9) do animal (Henneke et al., 1963). O peso excedente pode ter exercido compressão extra sobre o parênquima pulmonar, e, consequentemente, o retorno venoso prejudicado favoreceu o desenvolvimento da infecção pulmonar (López, 2012).

O uso do recurso radiográfico foi importante para confirmar as suspeitas clínicas e qualificar o diagnóstico das lesões vertebrais cervicais e a compressão medular entre $\mathrm{C}_{5}-\mathrm{C}_{6}$ (Hudson e Mayhew, 2005). As fraturas vertebrais visualizadas nas imagens radiográficas foram definidas na necropsia pela evolução do caso clínico, as ínfimas irregularidades nos corpos vertebrais observadas nas imagens radiográficas podem aventar possível mielite e as regiões radioluscentes foram confirmadas à necropsia como coágulos de sangue envolvendo o canal medular e a medular espinhal (Hudson e Mayhew, 2005).

Constatou-se, nos exames neurológicos, que a condução dos estímulos nervosos se reduzia diariamente. Provavelmente, a lesão e a 
compressão medular promoveram oxigenação deficiente, o que levou à isquemia, à hipóxia, ao edema com danos irreversíveis, à mielina e aos axônios (Steffen-Albert e Jeffeeson, 2012). Mediante prognóstico desfavorável do caso clínico, optou se pela eutanásia do animal, após consentimento do proprietário.

A realização de um exame neurológico minucioso e o conhecimento de neuroanatomia são de suma importância para caracterizar a localização de uma suposta lesão na coluna vertebral cervical. O diagnóstico definitivo pode ser confirmado associando-se avaliação radiográfica simples e contrastada. Com a descrição deste caso, é possível predizer que a dualidade desses exames é importante para o diagnóstico e o propósito a ser relevado na conduta terapêutica de um equino com fratura de vértebra cervical associada à compressão da medula espinhal.

\section{REFERÊNCIAS}

BIERVLIET, J.V.; SCRIVANI, P.V.; DIVERS, T.J. et al. Evaluation of decision criteria for detection of spinal cord compression based on cervical myelography in horses: 38 cases (19812001). Equine Vet. J., v.36, p.14-20, 2004.

BORGES, A.S.; SILVA, D.P.G.; GONÇALVES, R.C. et al. Fraturas vertebrais em grandes animais: estudo retrospectivo de 39 casos (19872002). Arq. Bras. Med. Vet. Zootec., v.55, p.127$132,2003$.

DE LAHUNTA, A.; GLASS, E.; KENT, M. Cerebrospinal Fluid and Hydrocephalus. In:_ Veterinary neuroanatomy and clinical neurology. 5.ed. Missouri: Saunders Elsevier, 2015. p.78-101.

HENNEKE, D.R.; POTTER, G.D.; KREIDER, J.L.; YEATS, B.F. Relationship between body condition score, physical, meansurements and body fat percentage in mare. Equine Vet. J., v.15, p.371-372, 1963.
HUDSON, N.P.H.; MAYHEW, I.G. Radiographic and myelographic assessment of the equine cervical vertebral column and spinal cord. Equine Vet. Educ., v.17, p.34-38, 2005.

LIN, C.Y.; WRIGHT, J.; BUSHNIK, T.; SHEM, $\mathrm{K}$. Traumatic spinal cord injuries in horseback riding: a 35-year review. Am. J. Sports. Med., v.39, p.2441-2446, 2011.

LÓPEZ, A. Sistema respiratório. In: ZACHARY, J.F.; MCGAVIN, M.D. Bases da patologia em veterinária. 5.ed. Rio de Janeiro: Elsevier, 2012. p.507-517.

MATTHEWS, H.K. Traumatismo espinhal, vertebral e intracraniano. In: REED, S.M.; BAYLY, W.M. Medicina interna equina. Rio de Janeiro: Guanabara Koogan, 2000. p.394-402.

MOROÓKA, M. Autocateterismo vesical intermitente técnica limpa: descrição do procedimento realizado pelos pacientes com lesão medular. Rev. Esc. Enferm., 2002. Disponível em: $<$ http://www.scielo.br/scielo.php?script=sci_artte xt\&pid $=$ S0080-62342002000400005 $>$. Acessado em: 28 jul. 2016.

RIET-CORREA, F.; RIET-CORREA, G.; SCHILD, A.L. Importância do exame clínico para o diagnóstico das enfermidades do sistema nervoso em ruminantes e equídeos. Pesqui. Vet. Bras., v.22, p.161-168, 2002.

SILVER, J.R. Spinal injuries resulting from horse riding accidents. Spinal Cord, v.40, p.264271, 2002.

STEFFEN-ALBERT, K.A.; JEFFEESON, T. Cuidados aos pacientes com traumas neurológicos. In: SMELTZER, S.C.; BARE, B.G.; HINKLE, J.L.; CHEEVER, K.H. Brunner \& Suddarth: tratado de enfermagem médicocirúrgica. 12.ed. Rio de Janeiro: Guanabara Koogan, 2012. p.1937. 